\title{
Enhancement of Surgical Training Practice with the Spring Tensor Heuristic Model
}

\author{
Christopher Chiu and Zenon Chaczko
}

\begin{abstract}
The enhancement of surgical simulation tools is an important research study, to assist in the assessment and feedback of medical training practice. In this research, the Spring Tensor Model (STEM) has been used for laparoscopic end-effector navigation through obstacles and high-risk areas. The modelling of the surgical trainer as part of the laparoscopic simulator seeks to emulate the physical environment as a virtualised representation in the integrated infrastructure. Combining sensor network framework paradigms to a surgical knowledge-based construct demonstrates how STEM can enhance medical practice. The architectural hybridisation of the training framework has enabled the adaptation of STEM modelling techniques for a simulated laparoscopic training methodology. The primary benefit of the architecture is that this integration strategy has resulted in a seamless transition of the heuristic framework to be applied to surgical training.
\end{abstract}

Keywords-laparoscopic surgical training, spring tensor model, modelling and simulation systems

\section{INTRODUCTION}

$\mathbf{T}$ HE development of a distributed sensor framework assists in the design of enhanced heuristic approaches to aid in medical training, without limiting the functionality of the surgical simulator tool. To achieve the design requirements, the prototype needs to incorporate the STEM heuristic to assist the supervisor of the surgical knowledge-based environment. The surgical knowledge-based framework includes the integration strategy and training information space, ensuring the system remains consistent with its primary responsibility to train surgeons on how to use laparoscopic tools effectively.

Autonomous training systems are examined by Rozenblit [1] as a means to provide efficient navigation and control in laparoscopic surgical scenarios. A particular concern is that the control strategies necessary to assess accuracy and performance [2] cannot be solved using traditional ArtificialIntelligence techniques. Given the progression of technological advances in wireless sensor technologies [3], [4], a distributed control approach using sensors combined with surgical training parameters for path coordination and neural networks for obstacle avoidance can be incorporated within the embedded system for monitoring and control. Thus, a multi-paradigm approach is employed to monitor the environmental surrounds of the end effector, while control aspects assist in surgical procedures and trajectory planning [5]. The use of a unified middleware framework to implement the controller functionality enables a uniform approach to coordinate the surgeon's

C. Chiu and Z. Chaczko are with the Faculty of Engineering and Information Technology, University of Technology, Sydney, Australia (e-mails: christopher.chiu@uts.edu.au; zenon.chaczko@uts.edu.au). activities; as well as provide localised awareness of obstacles in the vicinity of a regional zone [2].

As the end-effector is navigated to its desired destination, real-time feedback of obstacles enables the STEM heuristic to guide the trainee in mapping the global terrain for achieving the medical procedure [6]. This distributed approach to obtain a complete environmental map of the sensor's surroundings, provides redundancy in the situation where vital organs are positioned, or in the instance of different physiological contexts for a particular operation [7]. The research conducted by the University of Arizona [8] shows the growing concern about medical risks resulting from manual fatigue due to a lack of practice prior to new operational procedures being undertaken.

The research by Feng et al. [8] states the initiative of medical training systems to indicate the level of risk when conducting different surgical operations. The common types of errors in operations include miscounting of medical operation inventory, incorrectly identified procedures taking place and documentation errors. In another similar study conducted by Chuan et al. [9], the authors highlight the concerns of medical training procedures in multi-disciplinary health institutions in the United States. In this work, the focus is to develop a heuristic modelling approach that can reduce the rate of error by trainee surgeons, by highlighting the sensory factors that enhance health diagnosis concerns and provide rectification at the earliest opportunity.

The research from Feng and Chuan demonstrate that surgical training errors can occur during the operational procedure, with the effects resulting in life-threatening outcomes if the error is not detected at an early stage. Based upon the above discussion, the conclusion is that environmental perception can optimise the training process by augmenting the surgical process. The aim is to reduce the number of training errors introduced, ensuring that trainee surgeons perceive the vital statistics necessary to observe the quality of the procedure that they are conducting.

\section{SURgical Training ToOls Within HeURistic MODEL}

The actor-based paradigm as elaborated by the works of Georgeff et al. [10] has been employed to incorporate the rule-sets for the surgical training path manoeuvres, and tensoranalysis heuristics for obstacle avoidance [6]. In this system design, each software actor is associated with the sensors incorporated into the laparoscopic effector, and the motor controller mechanisms of the robotic effector when the surgical operation is conducted in a remote location. A core actor will 
represent the effector, such that a hierarchical structure of actor responsibilities exists within the medical training setup.

\section{A. Characteristics of Surgical Training Tools}

The actor-based characteristics defined in the model are elaborated as follows [4]:

- Central Gateway: The aggregation of statistical data from all medical sensors in a predefined region, and coordinate scheduled activities by the administrator or coordinator user:

- Health and Vital Life-sign Monitoring: Health monitoring data is collected from all sensors embedded in the laparoscopic effector, along with vital lifesigns of the patient imported from external data sources; such that it assists in forecasting potential health complications arising from a specific medical procedure that would affect trajectory path planning.

- Obstacle Mapping: Obstacle data accumulated from the end effector can be geo-tagged in a global map, thus allowing for the new mapping of undefined obstacles or refreshing existing obstacle maps for future medical procedures.

- Surgical End-Effector: The incorporation of various sensor data via a multi-modal approach [3], [11], communicating with neighbouring sensors in a peer-to-peer fashion, and centralising gateway exchanges for collaborative data exchange:

- Blood Pressure and Body Temperature: The monitoring of vital life-signs, along with additional data such as electrocardiogram measurements, will monitor localised health conditions and predict patient health stability throughout the medical operation.

- Triangulation Positioning: Sensors embedded with gyroscopic sensors provide a geographical reference point for accurate positioning of sensors relative to the patient's body; with the end-effectors incorporating static gyroscopes as designated anchor nodes.

- Ultrasonic and Infrared Sensors: For closeproximity obstacle detection so that pre-emptive path navigation can be established; additionally sensors can incorporate digital video capture with embedded machine vision algorithms for enhanced obstacle recognition.

Harnessing the surgical end-effector control dynamics as elaborated by the works of Chaczko et al [4], [5], the purpose is to examine the coordination of multiple craft irrespective of the medical health domain. The coordination and control of autonomous systems have its origins in autonomous robotic control [10], with the customisation in place to account for end-effector kinematic parameters. The main study of investigation is to determine what optimum method can be used to augment surgical training feedback in uncertain conditions.

\section{B. Ensuring Medical Diagnosis Optimisation}

The incorrect identification of obstacles or critical regions can lead to surgical errors and have life-threatening consequences [8]. This results from the misinterpretation of the procedure being conducted by hospital or ancillary staff. Thus, improper diagnosis can lead to the improper treatment of drugs supplied to a patient, or the wrong drug dosage as a result of an absence of required drug. For these reasons, the detection of defective medical diagnosis is critical.

\section{1) Obstacle Recognition}

The medical procedure needs to establish the potential obstacles or regions of interest before the operation takes place. The categorisation of potential obstacles by Chuan, Rozenblit et al. [9] is listed in the criteria below:

- Obstacle Geometry: The dimensions and shape of the obstacle can determine the criticality of the object during an operation. This means that while a smaller obstacle can be ignored, larger obstacles will result in a rerouted trajectory.

- Threshold Parameter Breaches: In case of breaching technical thresholds or parameters, the system must gracefully handle failure in such a way to minimise the occurrence of cascading failures during the operational procedure.

- Technical Defects: The operation must always ensure that all equipment is maintained to the highest quality. However, defects in the tensile strength and robustness of the end-effector can result in equipment failure. Therefore, the system must be able to detect minor deviations in performance that could indicate greater points of failure.

- Criticality of Region: Criticality of region provides an identity to distinguish zone of interest from the general surgical operation. This is important to ensure that only the required medical operation is conducted for the express purpose of treatment and operative care.

- Obstacle Identification: Each obstacle is uniquely identifiable to the end-effector depending on the operation performed. Impairment of the identification process can lead to errors in classifying obstacles.

- Obstacle Recognition: The obstacles throughout a procedure may vary depending upon the stage of operation. As an example, a blood vessel for a minor appendage must be distinguished from a major artery. Hence, it is important to recognise obstacles at an early stage throughout the medical procedure.

\section{2) Surgical Operation Concerns}

The surgical operation concerns identified from this research perspective include [1], [8]:

- Procedural Similarity: Similarity of procedures in terms of operational risk between medical domains, such as the entry point of a procedure being similar with one another;

- Equipment Usage: Resemblance of equipment and tools when operations are conducted in a medical scenario;

- Equipment Identification: Counting and identification of all medical tools used for the procedure, during and after the medical operation is completed; and

- System Quality: Ensuring all tools are accounted in terms of quality, with all contaminated equipment inspected, tested and sterilised before reuse. 
Referring to the abovementioned information leads to deduction that during an operational procedure, the risks associated with surgical operations can be addressed by augmenting the perception of the surgeon to highlight potential defects or risks throughout the medical task. The authors propose the use of the Spring Tensor analysis method to address these problems related with surgical training for global perception and awareness.

\section{Development Methodology of the STEM MODEL}

\section{A. Context of STEM with Surgical Training Dynamics}

The method to determine a globalized trajectory mapping method has been inspired by protein fluctuation dynamics. As discussed in depth by Lin and Song [6], the premise of the Spring Tensor Model (STEM) is to determine conformational changes in proteins using second-order partial derivatives as Hessians. Conformational change is the transition of macromolecular structures in proteins as a result in a change of acidity, temperature and voltages.

The spring tensor model is an enhancement of anisotropic modelling and Gaussian modelling methods, as while the former determines fluctuations of an atom's direction, the latter is better at determining the prediction of magnitudes of direction [6]. Thus by combining the two methodologies, the spring tensor model can be applied to a coordinated endeffector system as follows:

- Anisotropic Modelling: The determination of conformational variation or fluctuation in direction between elements.

- Adaptation: This is suitable for determining how the interactions between neighbouring end-effectors will result in the degree of directional fluctuation. The variation of potential direction will indicate what possible directions a sensor can travel if it is in proximity with a neighbouring sensor.

- Interpretation: Smaller anisotropic values indicate a smaller potential to alter the direction, while larger values indicate a larger potential to alter the direction.

- Gaussian Modelling: The determination of conformational variation or fluctuation in magnitude between elements.

- Adaptation: This is appropriate to ascertain how interactions between end-effectors will result in the magnitude or total range of the fluctuation. The variation of potential magnitude indicates the maximum range the sensor can travel towards.

- Interpretation: Smaller magnitudes values indicate a smaller potential to alter the distance, while larger magnitudes indicate a larger potential to alter the distance of the sensor.

In the application of the STEM Model by Lin and Song [6], the Go-like potential [12] is considered to take non-native and native conformations as the injected data; for this instance these values are the difference in the end-effector's Cartesian
TABLE I

Adaptation of STEM MOdEL TO SURgical Training

\begin{tabular}{ll}
\hline \hline Hessian Term & Adaptation to Surgical Context \\
\hline $\begin{array}{l}\text { Radius of } \\
\text { Connectivity }\end{array}$ & $\begin{array}{l}\text { Determined by the sum of the first term, } V_{1} . \\
\text { This value resolves the magnitude of change } \\
\text { between neighbouring objects in space. }\end{array}$ \\
$\sum_{\text {Bonds }} V_{1}\left(r, r_{0}\right)$ & $\begin{array}{l}\text { End effects between neighbouring objects is } \\
\text { observed in free space. An example is how the } \\
\text { impact of one object results in kinetic energy }\end{array}$
\end{tabular}

$$
\sum_{\text {Angles }}^{\text {Bond Angle }} V_{2}\left(\theta, \theta_{0}\right)
$$

- Determined by the sum of the second term, $V_{2}$. This value is used to calculate the direction of change between neighbouring objects.

- The angular displacement is the impact of change between objects is referred to the bond angle.

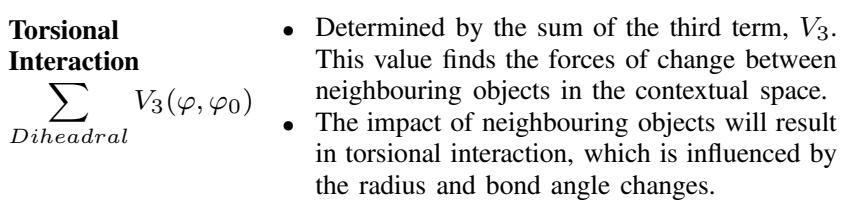

Torsional Interaction $\sum V_{3}\left(\varphi, \varphi_{0}\right) \quad$ neighbouring objects in the contextual space. $\sum_{\text {Diheadral }} V_{3}\left(\varphi, \varphi_{0}\right)$ - The impact of neighbouring objects will result in torsional interaction, which is influenced by

\begin{tabular}{|c|c|}
\hline $\begin{array}{l}\text { Non-Local / } \\
\text { Global } \\
\text { Interactions } \\
\sum_{i<j-3} V_{4}\left(r_{i j}, r_{0, i j}\right)\end{array}$ & $\begin{array}{l}\text { - Determined by the sum of the final term, } V_{4} \text {. } \\
\text { This term is of interest in this work, as it } \\
\text { determines the forces of change between non- } \\
\text { neighbouring objects. } \\
\text { - This value is important to the research as it } \\
\text { concerns how non-neighbouring objects can } \\
\text { impact the relations of all objects connected } \\
\text { in a shared field. } \\
\text { - The interpretation of these effects depending } \\
\text { on the medical context and domain. }\end{array}$ \\
\hline
\end{tabular}
the radius and bond angle changes.

coordinates between time $n$ and time $n+1$. These terms are divided into four terms as elaborated in Tab. I.

The unique value of epsilon and Taylor expansion parameters suitable for end-effector surgical contexts is obtained through experimental observation of the surgical sensors. In addition to their interaction in the physical environment, it is important to determine the thresholds of the end-effector's direction and magnitude to make a complex manoeuvre under various conditions, such as when a critical artery must be avoided to prevent medical complications during complex procedures. The minimisation of risk is an important factor in the success of the operation, once post-operative care and treatment is considered.

It is noted that there is no fixed parameter values that can be used for all end-effector technologies and devices, although a close approximation can be made for classes or category types of end-effectors that is sufficient for the majority of results. As an example of this property, a microincision end-effector will have a different operational action compared to a coarse-scale incision end-effector, due to their differentiation in size, weight and operational thresholds for each end-effector.

The surgical training environment by its nature will need to perform complex maneuvering tasks, due in part to the surgical 


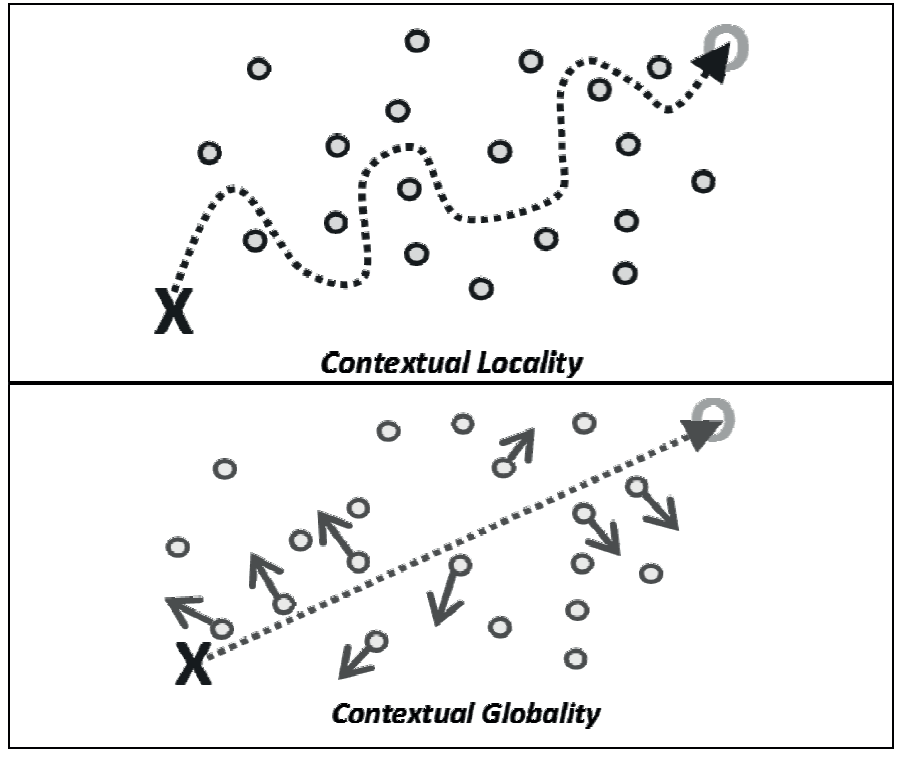

Fig. 1. Local vs. global context in free space [3].

operations that can be performed as a result in the advances in laparoscopic surgery [1], [8]. As a direct consequence of these medical advances, heuristic models need to reflect that the surgeon will be performing in more dynamic, unexpected environments with increasing complexity. The STEM heuristic model provides a method to visualize how local effects in the environment can impact on the global operational model.

The reason that these effects matter is because the expertise of the surgeon is his or her ability to navigate the end effector to the destination point, so that they can perform the operation with skill and expertise [1]. Operations vary in complexity, with some obstacles or critical zones being unavoidable, while other concerns are of lesser importance.

To provide a contextual perspective of critical zones, the skill of a laparoscopic surgeon is not just understanding how to use the end-effector in free space, but also his or her skill to recognize different surgical contexts and perform a maneuver based upon the operation being performed. As an example, a heart by-pass operation will involve more critical zones of risk compared to an appendix operation, due to a higher number of critical blood vessels and veins. Therefore, an optimum balance must be made between achieving an optimum end-effector trajectory, while simplifying the number of maneuvers made by the surgical operator.

The research exercise is to evaluate how sensor network heuristics can be applied to a laparoscopic surgical training platform, so that the operation is performed effectively and efficiently. The training environment must consider the importance of the end-effector sensing its surroundings and neighboring environment, so that auditing and feedback can take place. Feedback is essential for post-operational review and trajectory mapping analysis.

Trajectory mapping for surgical operations is an important task in training surgeons, as the simpler the maneuver, the risk of error made by the surgical trainee is reduced. As shown in Fig. 1 (Above) traditional trajectory mapping methods rely on

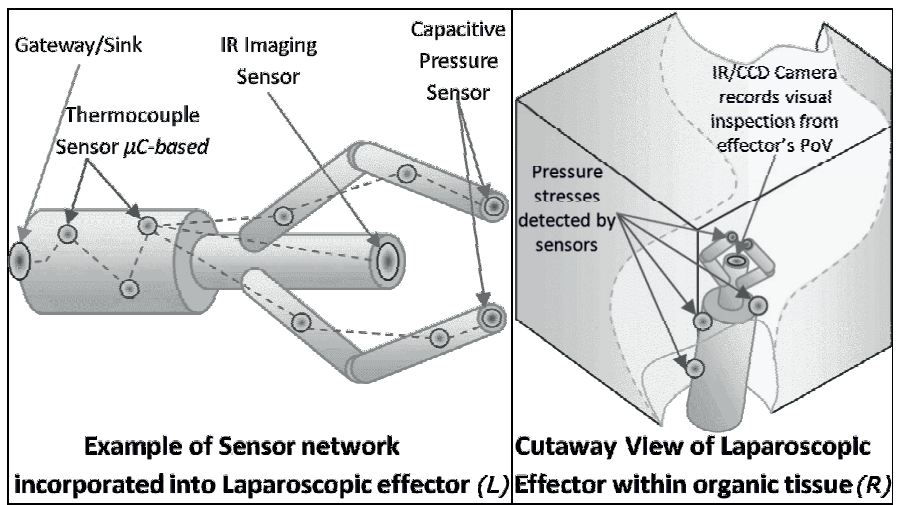

Fig. 2. Incorporation of sensors within the surgical training instrumentation [5].

local perception to maneuver around its neighbors, such as neural networks.

Such a trajectory path places emphasis on intelligence at the source, without realizing the bigger picture: that the path can be potentially complex and meandering. The environment is considered as a global perception, by taking a sensornetwork approach to the solution as shown in Fig. 1 (Below). This means that the neighboring environment is treated as an interconnected environment, such that the end-effector is not treated in isolation. Rather, the end-effector's movements have a direct and consequential impact to the environment around it. As an example, while damaging a small vein will have an inconsequential impact to the success of the operation, severing a main artery will result in major intervention by the surgical team.

\section{B. Development of the STEM Heuristic Platform}

The development of the STEM model for a Sensor-Actor network enabled laparoscopic surgical training tool assumes the consideration that includes maintaining an environment suitable for stable operational procedures. Thus, the training tools need to be verified in terms of their operational procedure or task prior to the surgical task taking place. As shown in Fig. 2, the system architecture needs to encapsulate the sensor network incorporated within the end-effector as the virtual surgical construct.

This ensures that the Vision System incorporated in the endeffector can be unified as part of the training exercises from the effector's Point of View (POV), so that the overall system can be quality controlled throughout the medical procedure. The synchronised recording of the procedure along with the medical sensory feedback is essential to provide real-time interactivity to the surgical trainee, so that they can enhance their skill and expertise in future training operations.

The machine vision for recording the medical procedure uses the existing Charge Coupled Device (CCD) camera built into the end-effector. The two-dimensional vision of the procedure uses a predefined sample template to sample the scale and variation of the operational procedure taking place, with identification of obstacles being relayed into the STEM model. 
TABLE II

PROCESSING APPROACH AND EXPERIMENTAL IMPACTS ON THE STEM MODEL

\begin{tabular}{|c|c|}
\hline $\begin{array}{l}\text { Processing } \\
\text { Approach }\end{array}$ & Impact on the Experimental Model \\
\hline $\begin{array}{l}\text { Direct } \\
\text { Interpretation }\end{array}$ & $\begin{array}{l}\text { Direct interpretation provides an injection of the raw } \\
\text { dataset into the STEM heuristic model: } \\
\text { - The first evaluation of the data structure con- } \\
\text { siders the direct the STEM model, with the } \\
\text { analysis of results being provided to the end } \\
\text { user. } \\
\text { - The direct instance considers the data structure } \\
\text { in its original form without any transforma- } \\
\text { tions or modifications of any kind. This allows } \\
\text { the heuristic to be evaluated purely on its } \\
\text { predictive capability. }\end{array}$ \\
\hline
\end{tabular}

Indirect

Transformation
Indirect transformation means that the data is processed according to existing geometric or other predefined rules, before it is processed by the STEM heuristic model as follows:

- The indirect approach incorporates a preprocessing stage and a main processing stage. The raw data set is optimised according to an objective function within the Particle Swarm Optimisation (PSO) heuristic.

- After the population is checked with the accepted fitness level, further manipulation of the data structure is halted. At this point, the transformed data structure is injected to the STEM model for final processing and analysis, with the results shown to the end user.
The Spring Tensor model considers all sensory devices as a coordinated network that is bound together by their connectivity [6], [11]. In STEM, the principle of connectivity is applied as a field of interconnected "springs", where the effects of one sensor result in the effect throughout the entire network structure. The process of optimising the STEM model depends upon the input parameters fed into the model, as an optimisation procedure to ensure that the results match against the desired level of fitness.

The structure of the data affects the validity and quality of the STEM results, with the combination of data structure optimisation techniques to improve classical global perception by reducing the overall computational complexity [5]. The use of STEM provides an optimisation tool to process large amounts of sensory information, as to determine the effects of change when the sensor network structure is impacted by localised changes in the surgical environment.

In Fig. 3, the process starts with initialisation of the raw data sets of obstacle positions and sensor node localisations. The experimental platform considers two main processing models, direct and indirect with the impacts on the experimental model as elaborated in Tab. II.

In this process, the STEM model processes the sensory and obstacle information as a unified instructional base, where both sensory data and obstacle data is handled equally in terms of data processing and priority. In order to reduce computational processing requirements, thresholds are established to display only significant changes in magnitude and direction above

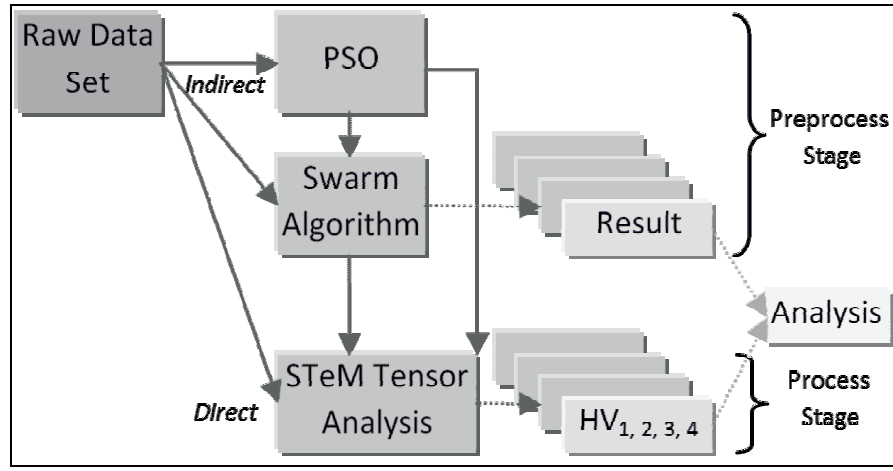

Fig. 3. Flow diagram for applying STEM to the surgical training scenario [3].

a pre-defined threshold. The current threshold is established to display all results within 3 Standard Deviations of the mean threshold result, or $99.7 \%$ of the result. The threshold parameters are customisable in the experimental model, and can be modified depending on the context of the evaluated medical domain.

The pre-processing stages in Fig. 3 are relevant when a data transformation methodology is applied to the STEM model. The premise of the experiment is to evaluate the impact of the data model when the Spring Tensor model is adopted to predict the magnitude and fluctuation of change in the medical training environment. The main source of laparoscopic training data is obtained from Chuan et al. [9].

\section{RESUlts OF EXPERIMENTAL STEM HEURISTIC}

The experiments were executed to determine the accuracy of the STEM model to predict the magnitude and direction of change for a simulated laparoscopic surgical procedure. The experimental model utilised the Virtual-Assisted Simulation Training (VAST) datasets obtained from the University of Arizona [8], with the data structures injected into the STEM source code adopted from Lin and Song [6]. The heuristic analysis was developed in MATHWORKS MATLAB 2012a, with Fig. 4 showing a sample surface plot of the $4^{\text {th }}$ Hessian of non-local contacts for a 60 -element dataset. The maximum value indicates the highest magnitude of change and direction occurring for the $38^{\text {th }}$ element in the input dataset.

Figure 5 shows the representation of the trajectory of change and magnitude in 3D space as directional arrows. The direction is indicated in the arrowhead, with the magnitude shown by the thickness and darkness of the arrow. Greater thickness and darkness indicate a stronger magnitude relative to each element being analysed in the STEM model. The user frontend environment depicts the direction of each element's longterm Hessian value in an isometric perspective within the sensor medical training environment.

The simulation environment will be extended in future to depict a more realistic surgical scenario, including the overlay of the critical organs and blood vessels that the surgeon will encounter in a real-life scenario. To handle the increased complexity for graphical modelling and simulation, a staged migration of the heuristic model to the VAST resource is necessary to facilitate further experimentation and 


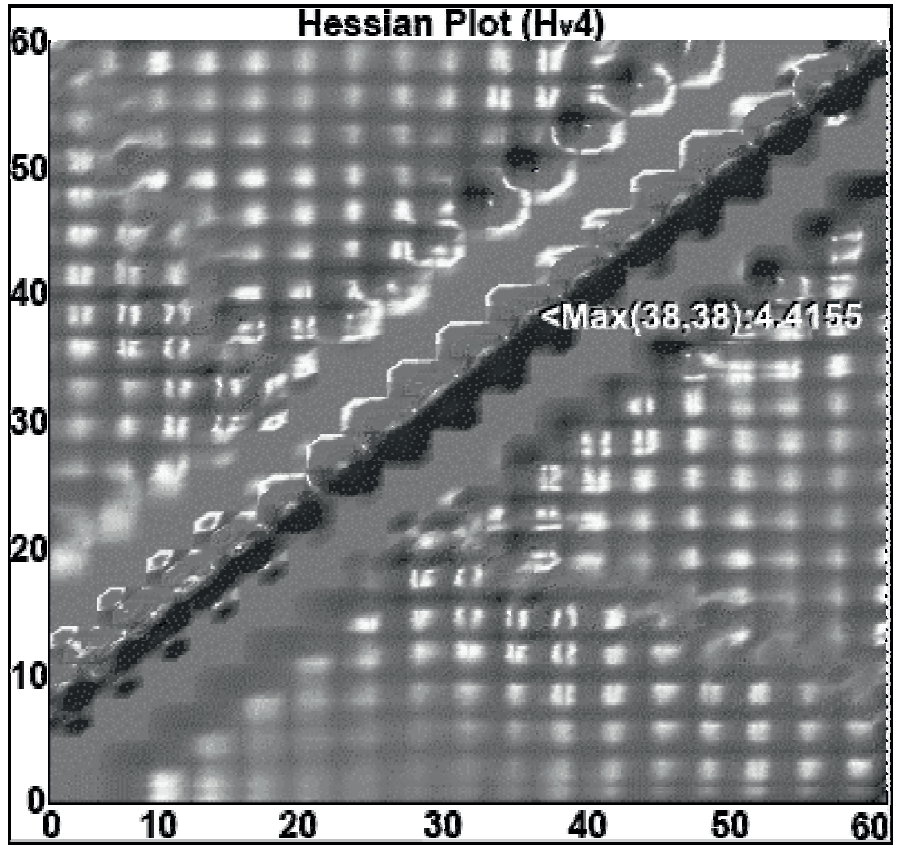

Fig. 4. Hessian surface plot of non-Local / global interactions (Hessian V4).

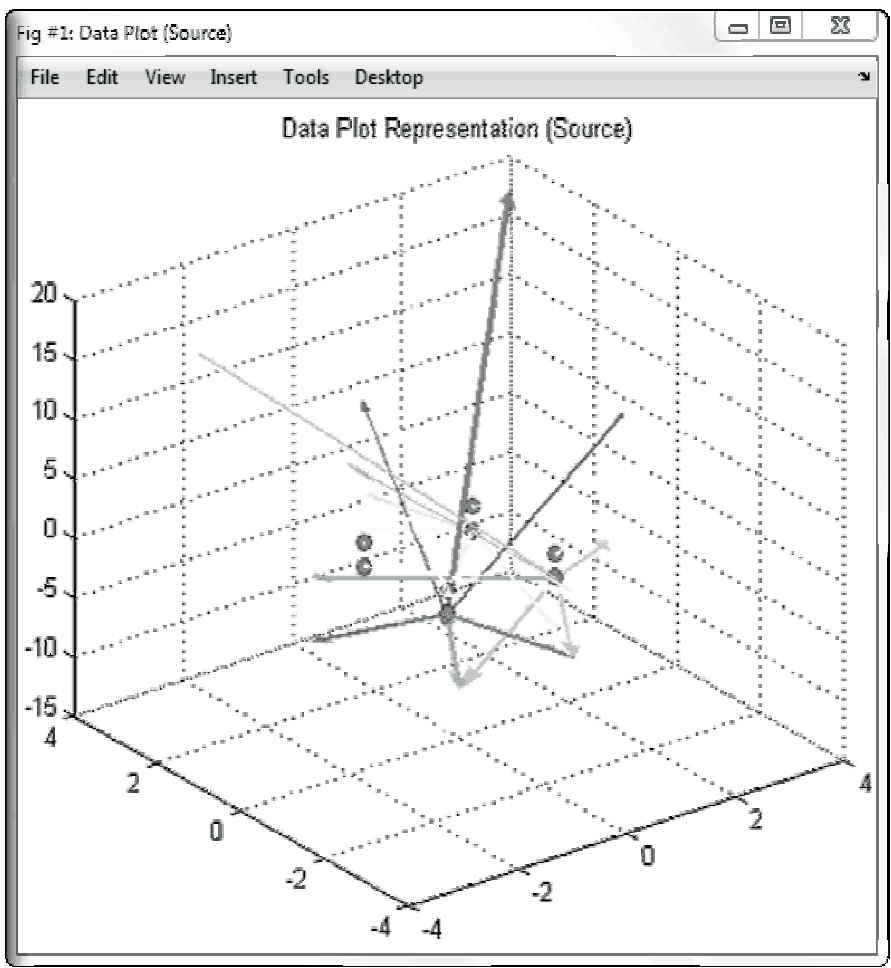

Fig. 5. Interpretation of Hessian plots as Cartesian points in 3D space.

analysis. The STEM heuristic has been designed so that it is programmatically portable, and it is not dependent upon any proprietary libraries or functions that will inhibit its use beyond the MATLAB development environment.

Furthermore, the surgical environment is designed to be progressively built upon the expertise of the surgical trainee, so that the heuristic analysis displayed to the trainers is suitable for the audience. As an example, while beginner surgeons will need to familiarise themselves with the equipment and learn how to navigate in free space, more advanced operators will learn navigational strategies and manoeuvres to operate in a safe manner. The STEM heuristic model can also be augmented in a similar manner. While the projection operation will be shown to all operators during the training exercise, a beginner operator will see just the major projections. Meanwhile, a more advanced operator will be able to see the minor projections as well to assist in training exercises that require greater precision.

The experimental approach conducted is categorised according into the dataset processing method used. This includes pseudo-randomised data, grid-lattice structured data and the Particle Swarm Optimisation approach as described in Tab. III.

As observed in Fig. 6, the purpose of the experiment is to measure the total number of projections made using the Tensor Analysis method. The results show that as the number of agents increase in the environment, so do the number of tensor projections. That is expected because the increase in population density will result in a larger number of interactions between sensors within the laparoscopic end effector. However, it is important to take note of the projection frequencies between random data sets: grid lattice structures having an average $15 \%$ lower difference of projections compared to random data, and PSO with an average $18 \%$ difference of projections compared to random data. As the purpose of utilising the STEM heuristic is to minimise the number of interactions of end-effector sensory devices interacting with other sensory devices, the results demonstrate that a PSO method is suitable to extend the heuristic process further for environmental global awareness.

The current results show that a combined heuristic modelling approach provides enhanced projection capability, compared to a single heuristic approach. Further experimentation is necessary to determine the performance effects of a multiheuristic strategy, and its ability to make projection operations in a near real-time manner. The suitability for a heuristic model to be applicable in medical contexts depends on its ability to handle continuous demands from the end-user; an ideal heuristic should be transparent to the operator and should not require external user intervention. In life-critical environments, real-time systems should have an extremely low failure rate, and rely on redundancy measures to minimise the potential of complete system failure.

Particle swarming in robotic contexts is based on the principle that the autonomous actuators all move in a particular direction, but for the context of surgical trajectory control this is not the desired behaviour. The current experimental model demonstrates how particle swarming can assist in global perception of the laparoscopic end-effector for a surgical scenario. It is noted that a process needs to be developed so that the swarming is executed as required; so an endeffector can execute a navigational path that is obscured by neighbouring obstacles. Further work needs to be developed to build a model that can balance the needs of the individual end-effector, while harnessing the cooperative capability of the virtual training environment using different metadata models. 
TABLE III

DATA MOdels USED FOR EXPERIMENTAL PlatForm

\begin{tabular}{ll}
\hline \hline $\begin{array}{l}\text { Data } \\
\text { structure }\end{array}$ & Adaptation to the experimental platform \\
\hline $\begin{array}{l}\text { Random Data } \\
\text { Generation }\end{array}$ & $\begin{array}{l}\text { Data is directly injected into the STEM model with no transformational process: } \\
\text { - The data is randomly generated using a Pseudo-Random algorithm (Mersenne- twister method) as a control baseline to } \\
\text { evaluate the other dataset layouts. } \\
\text { - The random data is subject to the limits of variation among the total population size using a predetermined threshold value. } \\
\text { - Random data provides the worst-case scenario, as the structure is not defined by any rules that govern its configuration. } \\
\text { - The use of the structure is an experimental control to determine the validity of alternate data structures. }\end{array}$
\end{tabular}

Grid-Lattice Data is indirectly transformed according to an equidistant grid lattice formation before being injected into the model:

Layout - The data is generated according to a grid lattice plane, with each element equidistant from each other.

- This data structure is used to evaluate how radial and linear trajectories can impact on geometrically layered structures, and its network impact resulting from geographically spread arrangements.

- Equidistance is based on the principle of geometric distribution, primarily on the physical plane of dimensionality.

- The free space of the experiment is defined in three-dimensions, in the X, Y and Z plane. The operation takes place according to a defined reference position, designated as the origin of the operational procedure.

\begin{abstract}
Particle
Swarm

Optimisation

Data is indirectly transformed according to Particle Swarm Optimisation heuristics before being injected into the model:

- The data is subject to Particle Swarm Optimisation (PSO) as described by the work of Clerc [13], with random, grid- lattice datasets and future data sources being processed by the PSO algorithm incorporated into the STEM model.

- The optimisation of the data to obtain a global minimum or maximum value is limited by the total number of iterations that can be executed, the objection function used and the thresholds values for inertia and correction factors.

- The purpose of utilising PSO is its ability to handle dimensions beyond the physical plane. This means that the metadata is not confined to the $\mathrm{X}, \mathrm{Y}$ and $\mathrm{Z}$ plane.

- To enhance the experimental model, additional data metrics are simulated in the experiment:

o The criticality of the obstacle encounter as a metric from 0 to 1 (highest being of most risk.)

o The signal strength of the wireless sensor from 0 to 100 (based on Signal to Noise ratio).

o The precision of the end-effector being utilised from 0 to 1 . A higher precision tool will have a greater threshold to perform in greater zones of risk, while a lower precision tool will have a reduced threshold to risk.
\end{abstract}

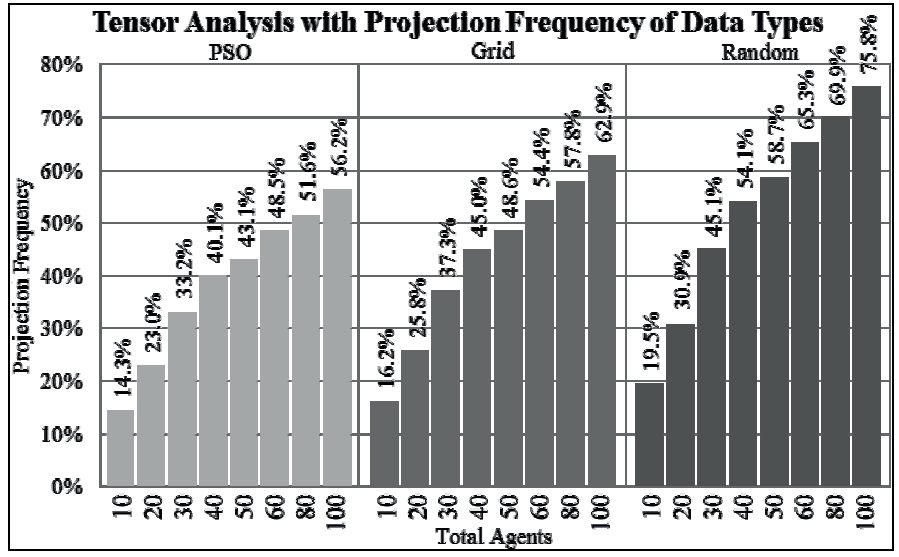

Fig. 6. Results of STEM analysis with projection frequency of different data types.

\section{CONCLUSION}

The incorporation of the STEM heuristic in a surgical training construct aims to assist the surgeon in determining the local impacts, which can cause global issues in the environmental field. A balance must be maintained between global optimality and local environmental awareness in the development of a surgical training system, by capturing the metric values necessary for projecting the magnitude and direction of change in the network. Using a multi-tiered approach to heuristics achieves the desire of achieving cooperative behaviour from a global context. This is because complete visibility of the network structure is maintained throughout the optimisation and coordination process, regardless of the trajectory path established in the sensory field. Although further research work is required to obtain parameter values that are suitable for analysing laparoscopic operations, the current results from the experimental prototype show potential in adopting the STEM heuristic for surgical training environments.

\section{REFERENCES}

[1] C. Feng, J. W. Rozenblit, A. J. Hamilton, and A. Wytyczak-Partyka, "Defining spatial regions in computer-assisted laparoscopic surgical training," in Proceedings of the 16th IEEE International Conference and Workshops on the Engineering of Computer Based Systems (ECBS), San Francisco, California, 2009, pp. 176-183.

[2] C. Feng, J. W. Rozenblit, and A. J. Hamilton, "A hybrid view in a laparoscopic surgery training system," in Proceedings of the 14th IEEE International Conference and Workshops on the Engineering of Computer Based Systems (ECBS), Tucson, Arizona, 2007, pp. 339-348.

[3] C. Chiu and Z. Chaczko, An Anticipatory Sanet Environment for Training and Simulation of Laparoscopic Surgical Procedures, Advanced Methods and Applications in Computational Intelligence: Topics in Intelligent Engineering and Informatics. Germany: Springer Media, 2012, ISSN 2193-9411.

[4] C. Chiu and Z. Chaczko, et al., "Sensor-Actor Network Solution for Scalable Ad-hoc Sensor Networks," Electronics \& Telecommunications Quarterly, International Journal of Electronics \& Telecommunications, vol. 58, no. 1, 2012, (KEIT-PAN 2012), ISSN 0867-6747. 
[5] Z. Chaczko, R. Klempous, J. Nikodem, and M. Nikodem, "Methods of sensors localization in Wireless Sensor Networks," in Proceedings of the 14th IEEE International Conference and Workshops on the Engineering of Computer Based Systems (ECBS), 2007, pp. 145-152.

[6] T.-L. Lin and G. Song, "Generalized spring tensor models for protein fluctuation dynamics and conformation changes," BMC Structural Biology, vol. 10, p. 12, 2010, doi: 10.1186/1472-6807-10-S1-S3.

[7] Z. Chaczko, J. Nikodem, R. Klempous, and M. Nikodem, "Sensor Localisation Methods in the COSA Framework," in 2nd Conference on Wireless Broadband and Ultra Wideband Communications (AusWireless), 2007, p. 60.

[8] C. Feng, J. W. Rozenblit, and A. Hamilton, "A computerized assessment to compare the impact of standard, stereoscopic and high-definition laparoscopic monitor displays on surgical technique," Surgical Endoscopy, vol. 24, no. 11, pp. 2743-2748, 2010, doi: 10.1007/s00464-010-1038-6.
[9] C. Feng and J. W. Rozenblit, et al., "Surgical training and performance assessment using motion tracking," in Proceedings of the 13th IEEE International Conference and Workshops on the Engineering of Computer Based Systems (ECBS), 2006, URL: http://dev.astec.arizona.edu/home.

[10] M. Georgeff and A. Lansky, "Procedural Knowledge," Proceedings of the IEEE (Special Issue on Knowledge Representation), vol. 74, pp. 1383-1398, 1986.

[11] Z. Chaczko, P. Moses, and C. Chiu, "Cooperative Extended Kohonen Mappings for Wireless Sensor Networks," Eurocast 2009 Revised Selected Papers: Lecture Notes in Computer Science, vol. 5717, no. 5717, 2009, Berlin, Germany: Springer Media.

[12] C. Clementi, H. Nymeyer, and J. N. Onuchic, "Topological and energetic factors: What determines the structural details of the transition state ensemble and en-route intermediates for protein folding?" Journal of Molecular Biology, vol. 298, pp. 937-953, 2000.

[13] M. Clerc, Particle Swarm Optimization. London, UK: ISTE, 2006. 